\title{
TURBULENCE IN MARKET DEMAND ON SUPPLY CHAIN NETWORKS
}

\author{
Cannella, S.; Dominguez, R. ${ }^{* *} \&$ Framinan, J. M.* \\ * Industrial Management \& Business Administration Department, School of Engineering, \\ University of Seville, Spain \\ ${ }^{* *}$ Centre for Management Studies, Instituto Superior Técnico, Technical University of Lisbon, \\ Portugal \\ E-Mail: cannella@us.es, roberto.dominguez@tecnico.ulisboa.pt,framinan@us.es
}

\begin{abstract}
Recent works have shown that the dynamic performance (i.e., the so-called bullwhip effect) of serial and divergent Supply Chain Networks (SCNs) under a sudden increase in the average customer demand may be substantially different. However, the effect of customer demand variability has not been investigated despite the fact that, in real-world business, a turbulence condition on the market usually generates an increase of customer demand variability. Herein we focus on this particular market condition by studying the dynamic behaviour of both serial and divergent SCNs under a sudden increase both in mean demand and variance. Results show how an isolated impulse in the variability of customer's orders results in the same bullwhip effect for both serial and divergent SCNs. From a managerial view point, this finding provides new interesting insights for SCN managers.

(Received in September 2015, accepted in April 2016. This paper was with the authors 2 months for 2 revisions.)
\end{abstract}

Key Words: Serial Supply Chains, Divergent Supply Chains, Agent-Based Simulation, Shock Demand, Demand Variability, Demand Impulse

\section{INTRODUCTION}

A common phenomenon shown by Supply Chain Networks (SCNs) is the so-called bullwhip effect [1], which refers to an increase of the variability of order in the upstream direction toward producers and suppliers [2]. SCN managers and researchers have paid a lot of attention on this phenomenon due to its importance in nowadays economy, [3, 4]. Several studies have been performed to improve the understanding of the causes and consequences of the bullwhip effect. Among these, a recent study by Dominguez et al. [5] compares the bullwhip effect between two different SCN configurations: serial vs. divergent/distribution (i.e. each company furnishes two downstream companies). SCNs are modelled by SCOPE, a Multi-Agent based simulation software. In their work, the authors adopt the framework described by Towill et al. [6] for analysing the bullwhip effect. This framework proposes three "observer's perspectives" to analyse the bullwhip effect, i.e.: Variance lens, Shock lens and Filter lens. Dominguez et al. [5] adopt two demand models according to the variance lens and the shock lens. The former uses a stationary input demand to infer on the response of SCNs, while the latter uses an unpredicted and serious alteration in the customer demand in order to assess the resilience of a SCN [7].

According to the authors, the computational results on demand amplification show little differences between both SCNs for a stationary demand. Nonetheless, a sudden increase in demand makes an important difference: the divergent SCN shows to be more sensitive to the forecast deviations caused by this serious alternation, resulting in a higher bullwhip effect and a higher time to recover stability, thus increasing SCN costs. A managerial consequence is that a greater attention to the demand amplification phenomenon has to be paid for arborescent SCNs in sectors characterized by an unstable final demand, as compared to classical serial SCNs. 
In order to emulate the stress test, Dominguez et al. [5], as suggested by Towill et al. [6], adopt a customer demand distribution $N\left(\mu, \sigma^{2}\right)$ which suffers an average increase of $100 \%$ at a certain point of the simulation time. However, under turbulent market conditions, it is foreseeable that the variance of the customer demand can analogously experiment a potential increase. In this fashion, the purpose of this work is to extend the findings of Dominguez et al. [5] by comparing the performance of a serial SCN and a divergent SCN under a shock in the variability of demand. To do so, we first replicate the same serial and divergent SCN structures proposed by the authors under identical parameter conditions and modelling approach. Secondly, we perform a new experiment in which we analyse the different response between the two structures under a market condition characterised by a sudden increase both in mean demand and variance. Results reveal how an isolated impulse in the variability of customer's orders does not lead to different levels of bullwhip effect for both types of SCNs.

The paper is structured as follows: Section 2 briefly presents the adopted methodology (SCOPE). Section 3 presents the SCN model. Section 4 provides a detailed presentation of the experiments and the simulation outputs. Section 5 presents a summary of findings. Finally, Section 6 presents the conclusions.

\section{METHODOLOGY AND MODEL VERIFICATION}

The SCNs have been modelled using SCOPE [8], a Multi-Agent based software conceived for the analysis of complex SCN structures. SCN simulation entails modelling a system that represents all or part of its structure $[9,10]$. The simulator has been developed using Swarm, a Java-based software for Multi-Agent Systems development. SCOPE has been benchmarked against results offered by other authors. More specifically, Dominguez and Framinan [8] compare the results obtained by SCOPE on a four-echelon serial SCN with those obtained by Chen et al. [11], Dejonckheere et al. [12] and Chatfield et al. [13], as it is shown in Table I. For further information on the multi-agent software platform and on the validation process see Dominguez and Framinan [8] and Dominguez et al. [5].

Table I: SCOPE verification; amplification of standard deviation of orders on a 4-stage SCN.

\begin{tabular}{|l|c|c|c|c|}
\cline { 2 - 5 } \multicolumn{1}{c|}{} & Retailer & Wholesaler & Distributor & Factory \\
\hline Chen et al. [9] & 1.89 & 3.57 & 6.74 & 12.73 \\
\hline Chatfield et al. [11] vs Chen et al. [9] & 1.90 & 3.59 & 6.70 & 12.84 \\
\hline SCOPE vs Chen et al. [9] & 1.90 & 3.53 & 6.66 & 12.58 \\
\hline \multicolumn{5}{|l|}{} \\
\hline Dejonckheere et al. [10] & 1.67 & 2.99 & 5.72 & 11.43 \\
\hline Chatfield et al. [11] vs Dejonckheere et al. [10] & 1.67 & 2.99 & 5.72 & 11.43 \\
\hline SCOPE vs Dejonckheere et al. [10] & 1.71 & 3.10 & 5.96 & 11.93 \\
\hline
\end{tabular}

\section{MODEL DETAILS}

Nomenclature of the SCN model and statistic metrics are presented in Table II.

The main characteristics of the SCN models, according to Dominguez et al. [5], are presented below:

- Customer's demand $(C, j)$ follows a normal distribution with mean $\mu_{C, j}$ and variance $\sigma_{C, j}^{2}$ estimated by $\bar{D}_{C, j}$ and $s_{C, j}^{2}$. 
Table II: Nomenclature.

\begin{tabular}{|c|c|c|c|}
\hline$i$ & Echelon position in the SC & $\sigma_{L_{i j}}^{2}$ & Variance of the lead time of node $(i, j)$ \\
\hline$j$ & Node position in echelon $i$ & $s_{L_{i j}^{t}}^{2}$ & Estimated variance of the lead time of node $(i, j)$ \\
\hline$t$ & Time period & $R$ & Review period \\
\hline$K$ & Total number of echelons & $X_{i j}^{t}$ & Demand received by node $(i, j)$ during the "protection period" \\
\hline$C_{j}$ & Customer demand at position $\mathrm{j}$ & $\bar{X}_{i j}^{t}$ & $\begin{array}{l}\text { Estimated average of demand received by node }(i, j) \text { during the } \\
\text { "protection period" }\end{array}$ \\
\hline$\mu_{C, j}$ & Average demand placed by $C_{j}$ & $s_{X_{i j}^{t}}^{2}$ & $\begin{array}{l}\text { Estimated variance of demand received by node }(i, j) \text { during the } \\
\text { "protection period" }\end{array}$ \\
\hline $\bar{D}_{C, j}$ & Estimated average of demand placed by $C_{j}$ & $S_{i j}^{t}$ & Desired level of stock of node $(i, j)$ \\
\hline$\sigma_{C, j}^{2}$ & Variance of demand placed by $C_{j}$ & $z$ & Safety factor for the OUT policy \\
\hline$s_{C, j}^{2}$ & Estimated variance of demand placed by $C_{j}$ & $\tau$ & Moving averages and variance forecast period \\
\hline$D_{i j}^{t}$ & Demand faced by node $(i, j)$ & $\Phi_{i}$ & Order Rate Variance Ratio at echelon $i$ \\
\hline $\bar{D}_{i j}^{t}$ & $\begin{array}{l}\text { Estimated average of demand faced by } \\
\text { node }(i, j)\end{array}$ & $\sigma_{O_{i j}}^{2}$ & Variance of orders placed by node $(i, j)$ \\
\hline$s_{D_{i j}^{t}}^{2}$ & $\begin{array}{l}\text { Estimated variance of demand faced by } \\
\text { node }(i, j)\end{array}$ & $s_{O_{i j}^{t}}^{2}$ & Estimated variance of orders placed by node $(i, j)$ \\
\hline$O_{i j}^{t}$ & Orders placed by node $(i, j)$ & $\sigma_{d}^{2}$ & Variance of market demand \\
\hline$L_{i j}^{t}$ & Lead time of node $(i, j)$ & $s_{d}^{2}$ & Estimated variance of market demand \\
\hline$\mu_{L_{i j}}$ & Average lead time of node $(i, j)$ & $B w S l$ & Bullwhip slope \\
\hline $\bar{L}_{i j}^{t}$ & Estimated average lead time of node $(i, j)$ & $p_{i}$ & Position of the $i^{\text {th }}$ echelon \\
\hline
\end{tabular}

- The lead time of each node $(i, j) L_{i j}^{t}$ is independent from the rest of the nodes, with mean $\mu_{L_{i j}}$ and variance $\sigma_{L_{i j}}^{2}$, estimated by $\bar{L}_{i j}^{t}$ and $s_{L_{i j}^{t}}^{2}$ respectively. These lead times are gammadistributed with mean 4 units and c.v. $=0.50$. Nodes control the level of inventories using an Order-Up-To (OUT) policy with a review period of 1 unit.

- The demand received by node $(i, j)$ during the "protection period" $\left(L_{i j}^{t}+R\right)$ is denoted by $X_{i j}^{t}$ and estimated by $\bar{X}_{i j}^{t}$ and $s_{X_{i j}^{t}}^{2}$. Denoting by $D_{i j}^{t+k}$ the demand received by node $(i, j)$ at time $t+k, X_{i j}^{t}$ is computed as:

$$
X_{i j}^{t}=\sum_{k=0}^{L+R} D_{i j}^{t+k}
$$

- The OUT level, $S_{i j}^{t}$, allows to meet the demand during the time period $L_{i j}^{t}+R$ :

$$
S_{i j}^{t}=\bar{X}_{i j}^{t}+z S_{X_{i j}^{t}}
$$

Using this policy, at the beginning of every period $t$ each node places an order to raise or lower the inventory position to $S_{i j}^{t}$. In order to ensure a service level of $97.72 \%$ we use a safety factor of $z=2$ (Chatfield et al. [11]). When updating the OUT level $\left(S_{i j}^{t}\right)$, past demand and lead time data are available to nodes. This data are used to estimate the demand (mean, $\bar{D}_{i j}^{t}$ and variance, $s_{D_{i j}^{t}}^{2}$ ), and lead time mean $\left(\bar{L}_{i j}^{t}\right)$ at time period $t$. Finally, forecasts of lead-time demand mean $\bar{X}_{i j}^{t}$ and variance $s_{X_{i j}^{t}}^{2}$ are computed as in (3) and (4), respectively:

$$
\begin{aligned}
\bar{X}_{i j}^{t} & =\left(\bar{L}_{i j}^{t}+R\right) \bar{D}_{i j}^{t} \\
s_{X_{i j}^{t}}^{2} & =\left(\bar{L}_{i j}^{t}+R\right) s_{D_{i j}^{t}}^{2}
\end{aligned}
$$


The pair $\left(\bar{D}_{i j}^{t}, s_{D_{i j}^{t}}^{2}\right)$ are estimated by a $\tau$-period moving averages $(N P M A(\tau))$ and a $\tau$-period moving variances $(N P M V(\tau))$, respectively. According to Chatfield et al. [11], $\tau=15$ is used to compute $\left(\bar{D}_{i j}^{t}, s_{D_{i j}^{t}}^{2}\right)$, and an "all-data" approach $(\tau=t)$ to compute $\bar{L}_{i j}^{t}$.

- Negative replenishment orders are allowed.

- There is no collaboration between nodes of the SCN. Thus, each node's information is coming from the incoming demand flow (downstream members) and the outgoing flow of orders (upstream members).

- The sequence of actions to be performed by each node in each time period is as follows:

1. The OUT level $\left(S_{i j}^{t}\right)$ is updated adopting the forecast calculated in the previous period.

2. An order $\mathrm{O}_{\mathrm{ij}}^{\mathrm{t}}$ is placed to update the inventory position according to $S_{i j}^{t}$.

3. Goods are received from the upstream node.

4. Demand from downstream nodes $D_{i j}^{t}$ is received and satisfied.

5. A new forecast is calculated for the next period.

Nodes perform this sequence of actions from downstream echelons to upstream echelons.

\section{DESIGN OF EXPERIMENT AND SIMULATION OUTPUT}

To assess the bullwhip effect we use the same two metrics as in Dominguez et al. [5]. The first metric, Order Rate Variance Ratio $\left(\Phi_{i}\right)$ is a commonly-used metric for the bullwhip effect [12], and is computed (see Eq. (5)) as the ratio of the order variance of node $j$ in echelon $i\left(\sigma_{O_{i j}}^{2}\right.$, estimated by $\left.s_{O_{i j}}^{2}\right)$ to the order variance of the market demand ( $\sigma_{d}^{2}$, estimated by $s_{d}^{2}$ ). This metric can be adapted to a divergent SCN by using an aggregate measure for each echelon. If we assume that all customer demands are independent, the aggregate variance in each echelon $i$ can be approximated by the sum of the variances of orders of each node $j$ in echelon $i$, estimated by $\left(s_{O_{i j}}^{2}, s_{O_{C j}}^{2}\right)$. Using this approximation, $\Phi_{i}$ is formulated as in Eq. (6).

The second metric is a network measure called Bullwhip Slope $(B w S l)$. It is calculated as the slope of the linear interpolation of all ratios $\Phi_{i}$ (Eq. (7)). This metric allows an easy and efficient comparison between different SCNs, being an important indicator of bullwhip and inventory stability [14]. These metrics are summarized in Table III.

Table III: Metrics used for measuring the bullwhip effect.

\begin{tabular}{|c|c|c|c|}
\hline & Serial SCN & Divergent SCN & \\
\hline Order Rate Variance Ratio & $\Phi_{i}=\frac{s_{O_{i}}^{2}}{s_{d}^{2}}$ & $\Phi_{i}=\frac{\sum_{j=1}^{n_{i}} s_{O_{i j}}^{2}}{\sum_{j=1}^{n_{C}} s_{O_{C j}}^{2}}$ & (6) \\
\hline Bullwhip Slope & $\begin{array}{l}\qquad B w S l=\frac{K \sum_{i=1}^{K} p_{i}}{K \sum_{i=}^{K}} \\
K \text { is the total number of echelons }\end{array}$ & $\begin{array}{l}\frac{p_{i} \sum_{i=1}^{K} \Phi_{i}}{\left.K_{i=1} p_{i}\right)^{2}} \\
\text { position of the } i^{\text {th }} \text { echelon. }\end{array}$ & (7) \\
\hline
\end{tabular}

The demand distribution used by Dominguez et al. [5] for each customer is a $N\left(50,20^{2}\right)$ which experiments an increase of $100 \%$ in average when half of the simulation time has been run (excluding the warm-up period), turning into a $N\left(100,20^{2}\right)$. The results obtained by Dominguez et al. [5] for this scenario (here named $\lambda_{0}$ ) showed clear differences in performance between the two considered SCNs. The divergent SCN showed an increase in $\Phi$ of $95.86 \%$ over the serial SCN at the factory stage, and an overall increase of the bullwhip effect (summarized by $B w S l$ ) of $94.62 \%$. However, this study assumed that demand 
variability remained constant, thus obtaining a decrease in the relative variability (given by the coefficient of variation (c.v.)). More specifically, after the shock in demand a $50 \%$ reduction of the demand c.v. is experienced:

$$
\text { c. } v \text {. }(\text { before shock })=20 / 50=0.4 \rightarrow \text { c. } v \text {. }(\text { after shock })=20 / 100=0.2
$$

In this study we assume that a sudden increase in the mean demand due to market offers or economic staff would also lead to an increment in demand variance, maintaining a similar relative tendency (similar coefficient of variation). Thus, we present an analysis of this particular effect by comparing the results obtained from the following three scenarios:

- $\lambda_{0}$ : The original scenario (c.v. $0.4 \rightarrow 0.2$ ).

- $\lambda_{1}$ : This scenario analyses the performance under a $100 \%$ increase in the mean of customer orders, and a $50 \%$ in the standard deviation of customer demand (c.v. $0.4 \rightarrow 0.3$ ).

- $\lambda_{2}$ : This scenario is characterized by a $100 \%$ increase in the mean of customer orders, and a $100 \%$ increase in the standard deviation of customer orders (c.v. $0.4 \rightarrow 0.4$ ).

The details of this set of experiments are reported in Table IV. Results obtained for $\Phi$ are graphically represented in Fig. 1, and numeric results obtained for $B w S l$ are summarized in Table V.

Table IV: Sensitivity to demand variability: set of experiments.

\begin{tabular}{|c|c|c|c|}
\hline Experiment & Demand Pattern & Structure of the SCN & Metrics \\
\hline$\lambda_{0}$ & $\begin{array}{l}N\left(50,20^{2}\right) t \in[0-449] \\
N\left(100,20^{2}\right) t \in[450-700]\end{array}$ & \multirow{3}{*}{$\begin{array}{c}\text { Serial \& Divergent } \\
\text { SCNs }\end{array}$} & \multirow{3}{*}{$\begin{array}{c}\Phi ; B w S l \\
t \in[450-700\end{array}$} \\
\hline$\lambda_{1}$ & $\begin{array}{l}N\left(50,20^{2}\right) t \in[0-449] \\
N\left(100,30^{2}\right) t \in[450-700]\end{array}$ & & \\
\hline$\lambda_{2}$ & $\begin{array}{l}N\left(50,20^{2}\right) t \in[0-449] \\
N\left(100,40^{2}\right) t \in[450-700]\end{array}$ & & \\
\hline
\end{tabular}

The results in Fig. 1 show that $\Phi$ decreases, particularly at upstream stages of both SCNs (Distributor and Factory) as the variability of customer orders increases within the combined impulse in customer demand. In addition, the opposite trend is more severe in the divergent SCN. As a consequence, the differences in $\Phi$ between the serial and the divergent SCNs are reduced under a combined impulse (mean and variability) in the customer demand, and these differences are lower as the sudden increase in demand variability is higher.

$\lambda_{0}$

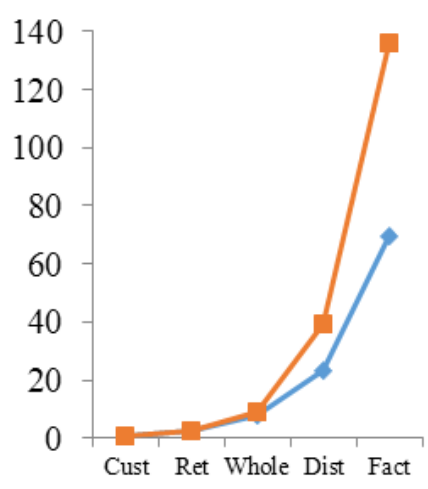

$\lambda_{1}$

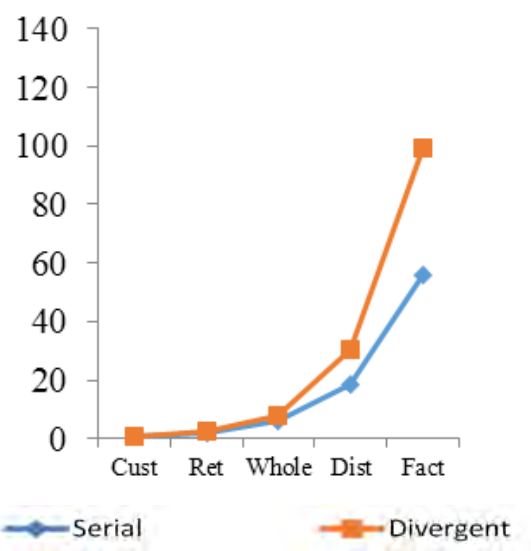

$\lambda_{2}$

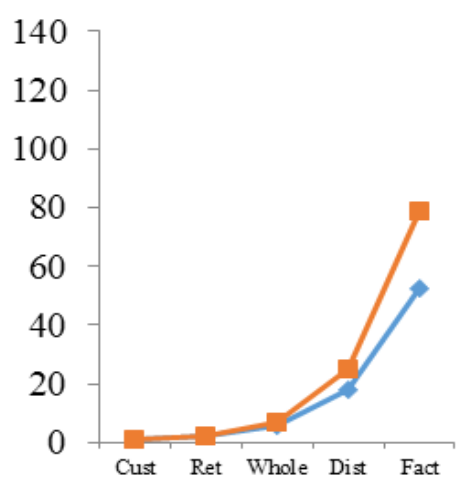

Figure 1: $\Phi$ under a combined impulse in mean and standard deviation of customer orders. 
The above results are confirmed by the $B w S l$ (Table V). It can be noticed that an increase in the impulse of demand variability $\left(\lambda_{0} \rightarrow \lambda_{2}\right)$ leads to a $24.95 \%$ reduction of the $B w S l$ in the Serial SCN and to a higher reduction $(41.89 \%)$ in the Divergent SCN. Also, differences between both SCNs decrease by shifting from $\lambda_{0}(94.62 \%)$ to $\lambda_{2}(50.72 \%)$.

Overall, these results might suggest that an impulse in the standard deviation of customer orders generates an enhancement of the process performance within both SCNs. Moreover, it seems that the performance of both $\mathrm{SCNs}$ becomes closer, thus reducing the differences between serial and divergent SCNs. This behaviour induces to think that an impulse in the variability of customer demand acts as a bullwhip deterrent, nevertheless this elucidation is essentially false due to the inherent properties of $\Phi$.

Table V: BwSl under a combined impulse in mean and standard deviation of customer orders.

\begin{tabular}{|c|c|c|c|}
\cline { 2 - 4 } \multicolumn{1}{c|}{} & $\begin{array}{c}\boldsymbol{B} w \boldsymbol{S l} \\
\text { Serial SCN }\end{array}$ & $\begin{array}{c}\boldsymbol{B} w \boldsymbol{S l} \\
\text { Divergent SCN }\end{array}$ & $\begin{array}{c}\boldsymbol{B} w \boldsymbol{S l} \text { increase } \\
\text { Serial SCN }->\text { Divergent SCN }\end{array}$ \\
\hline$\lambda_{0}$ & 15.79 & 30.73 & $\mathbf{9 4 . 6 2} \%$ \\
$\lambda_{1}$ & 12.56 & 22.48 & $\mathbf{7 8 . 9 8} \%$ \\
$\lambda_{2}$ & 11.85 & 17.86 & $\mathbf{5 0 . 7 2} \%$ \\
\hline $\begin{array}{c}B w S l \text { reduction } \\
\left(\lambda_{0} \rightarrow \lambda_{2}\right)\end{array}$ & $\mathbf{2 4 . 9 5 \%}$ & $\mathbf{4 1 . 8 9 \%}$ & \\
\hline
\end{tabular}

By analysing the standard deviation of orders at the producer stage of the divergent SCN, bullwhip increases as the variability of customer orders increase, under the same impulse in mean demand (see Table VI). However, the increases are not proportional: $\sim 50 \%$ and $\sim 100 \%$ increases in the standard deviation at customer stage correspond to $\sim 26 \%$ and $\sim 50 \%$ increases in the standard deviation of orders at factory stage, respectively. Consequently, since $\Phi$ at the factory level is computed as the ratio between the variance of orders placed by this echelon and the variance of the customer demand, the above mentioned behaviour generates the observed reduction in $\Phi$. We argue that this peculiar behaviour is due to the combined impulse in variance and mean of customer orders. Even though the magnitude of the variance of the factory stage is due to combined effect of the impulse in mean demand and impulse in variance demand, this result suggest that the effect of the impulse in the mean demand is dominant over the effect of the impulse in demand variability. For these reasons, by shifting from scenario $\lambda_{0}$ to scenario $\lambda_{2}, \Phi$ decreases.

Table VI: Combined impulse in customer demand mean and standard deviation of orders (divergent SCN).

\begin{tabular}{|c|c|c|}
\hline Scenario & Standard deviation of customer orders & Standard deviation of factory orders \\
\hline$\lambda_{0}$ & 57.53 & 671.42 \\
$\lambda_{1}$ & 84.85 & 845.69 \\
$\left(\lambda_{0} \rightarrow \lambda_{1}\right)$ & $\mathbf{4 7 . 4 9 \%}$ & $\mathbf{2 5 . 9 6 \%}$ \\
$\lambda_{2}$ & 113.05 & 1004.76 \\
$\left(\lambda_{0} \rightarrow \lambda_{2}\right)$ & $\mathbf{9 6 . 5 1 \%}$ & $\mathbf{4 9 . 6 5 \%}$ \\
\hline
\end{tabular}

In order to verify the above mentioned dynamics, we isolate the effects of impulse in the standard deviation of customer orders. Thus, we simulate a further experiment for which the mean of the customer demand remains unaltered over the entire simulation time, and the standard deviation of customer orders increases by $50 \%$ at $t=450\left(\lambda_{3}\right)$, and compare it with the NO-impulse scenario. Table VII shows the results of this experiment for the divergent 
SCN. It can be noticed that the variability of orders placed by the factory increases proportionally to the increases in the variability of orders placed by customers. Hence, $\Phi$ remains unaltered under this typology of impulse and is identical for both SCNs, as shown in Fig. 2.

Table VII: Effects of an impulse in standard deviation of customer orders (divergent SCN).

\begin{tabular}{|c|c|c|}
\hline Scenario & $\begin{array}{c}\text { Standard deviation of } \\
\text { customer orders }\end{array}$ & $\begin{array}{c}\text { Standard deviation of } \\
\text { factory orders }\end{array}$ \\
\hline NO-impulse & 55.79 & 433.12 \\
$\lambda_{3}$ & 79.34 & 620.60 \\
$\left(\right.$ NO-impulse $\left.\rightarrow \lambda_{2}\right)$ & $\mathbf{4 2 . 2 1 \%}$ & $\mathbf{4 3 . 2 9 \%}$ \\
\hline
\end{tabular}

$\Phi$ NO-impulse scenario

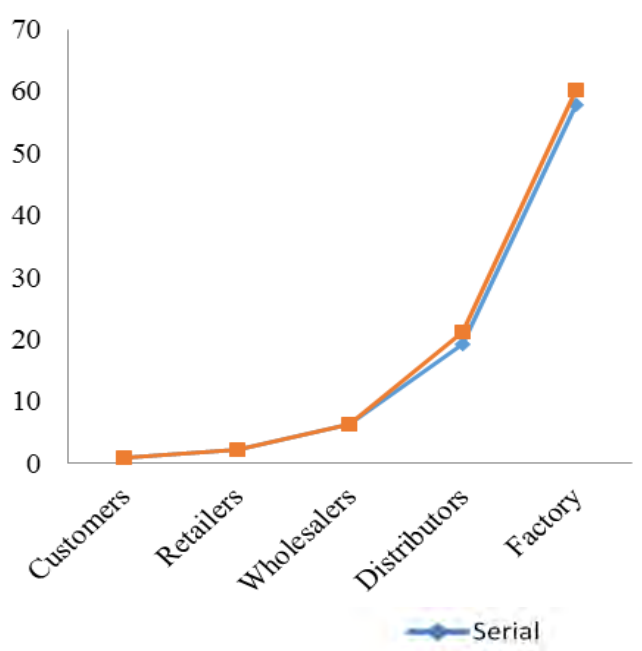

$\Phi \lambda_{3}$ scenario

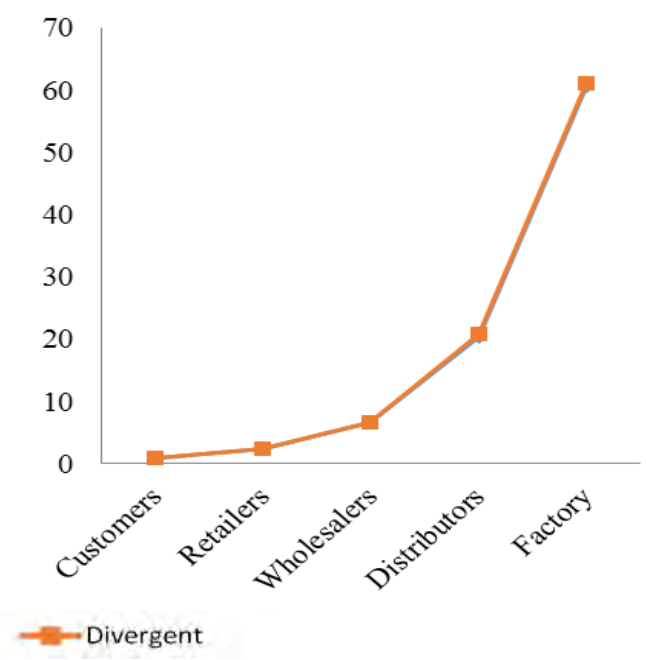

Figure 2: A comparison of $\Phi$ between the NO-impulse scenario and an impulse in the variability of customer orders.

\section{FINDINGS}

In this section, we summarize the findings of this work according to the typology of impulse demand.

\subsection{Combined impulse of customer's demand mean and variability}

This scenario involves a combined and sudden impulse in customer's demand mean and variability. In this case, since the effect of the impulse in demand mean is the main cause of the increase of the order variability, we can expect a general increase of $\Phi$. However, due to the dominance of the effect of the impulse on demand mean over the effect of the impulse on demand variability, for a given impulse on demand mean we obtain lower values of $\Phi$ as the impulse in customer's demand variability increases.

Thus, in case of a combined impulse in customer's demand, a complementary metric of the bullwhip effect that is able to focus on the dominant effect is needed. We propose to use a Peak of Orders Ratio (POR), which is a good measure to account for the extreme swings in order patterns of each echelon [6]:

$$
P O R_{i}=\frac{\max O_{i}^{T}-\min O_{i}^{T}}{\max O_{C}^{T}-\min _{C}^{T}}
$$


We use this metric to compare scenarios $\lambda_{0}$ and $\lambda_{1}$ for the factory stage of the divergent SCN, obtaining $P O R_{\text {factory }}\left(\lambda_{0}\right)=12.29$ and $P O R_{\text {factory }}\left(\lambda_{1}\right)=12.58$. According to this metric, the factory of the divergent SCN shows a very similar reaction to the customer's impulse in demand in both scenarios, showing a slightly higher turbulence in scenario $\lambda_{1}$ than in scenario $\lambda_{0}\left(P O R_{\text {factory }}\left(\lambda_{1}\right)>P O R_{\text {factory }}\left(\lambda_{0}\right)\right)$. In order to better appreciate this phenomenon, we plot in Fig. 3 the order quantities placed by the factory of the divergent SCN in both scenarios. In fact, factory's order patterns are very similar during a short time after the impulse in customer demand (reaction to the shock), being slightly more nervous in scenario $\lambda_{1}$, as reported by the $P O R_{\mathrm{i}}$ metric. When the factory is recovered from the shock, it can be seen how the order pattern in scenario $\lambda_{1}$ shows a higher variability (since customer demand has increased its variability) than the order patter in scenario $\lambda_{0}$. Thus, $P O R_{\mathrm{i}}$ is a good metric to be used together with $\Phi$ in order to analyse the bullwhip effect in this kind of scenarios.

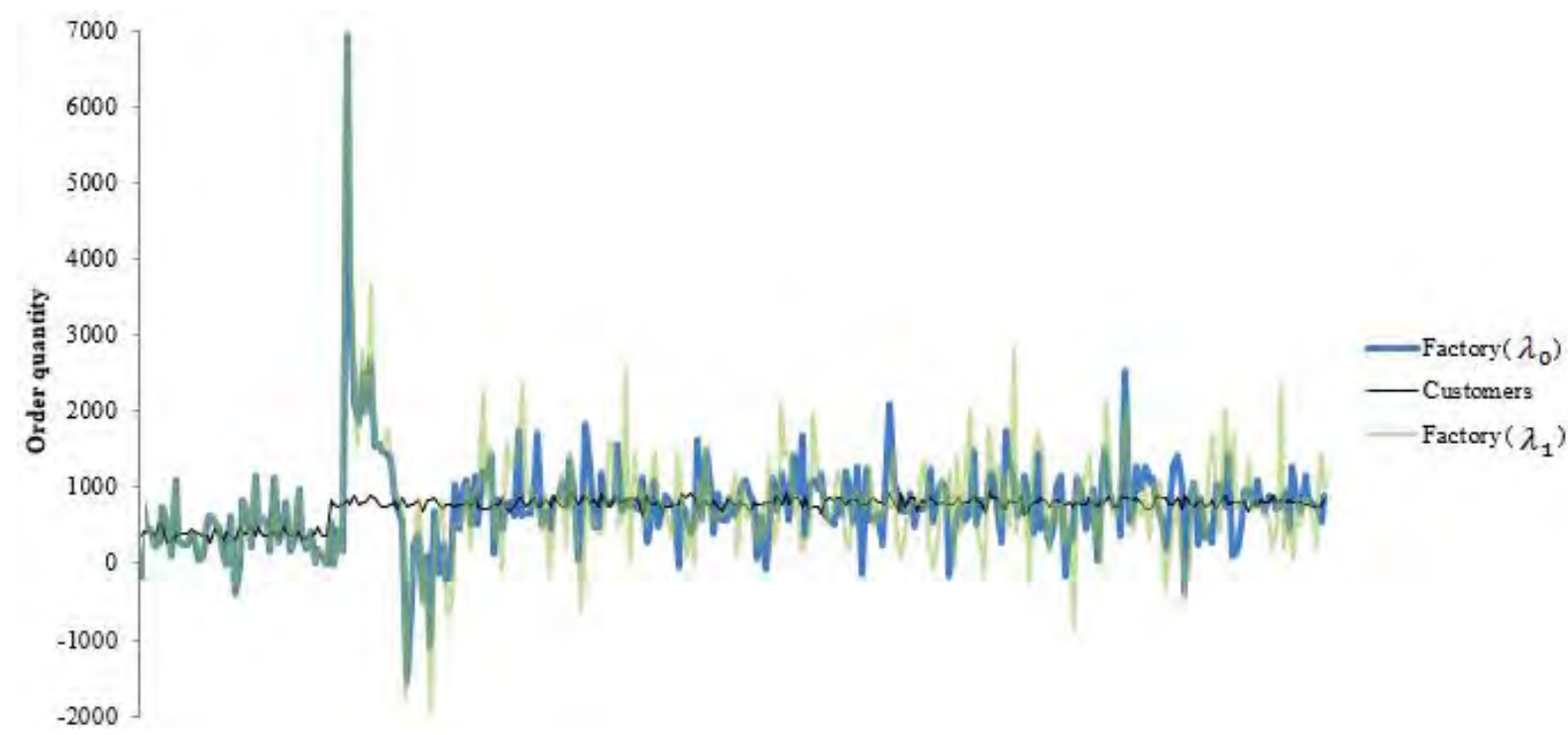

Figure 3: Factory's order pattern in the divergent SCN in different scenarios.

\subsection{Isolated impulse of customer's demand mean and customer's demand variability}

Unlike the previous scenario, results show how, under a sudden increase in demand variability, all stages of both SCNs react by proportionally increasing the variability of their orders. In this fashion, both SCNs reveal an identical behaviour. On the contrary, a sudden and severe impulse in customer's demand mean, as described by Dominguez et al. [5] generates a discrepancy between the performances of the two SCNs. In this case, since the variability of customer demand remains constant during the impulse, the effect of the impulse in the customer's demand mean is well summarized by $\Phi$. As reported by Dominguez et al. [5], important dynamic effects can be expected, obtaining a high increase in the bullwhip effect and important differences in the performance of both SCNs.

\section{CONCLUSIONS}

This study completes and extends the results presented in Dominguez et al. [5] regarding the impact of customer's demand variability on a shock demand on different SCNs. We have compared the bullwhip effect observed in a serial SCN with the bullwhip effect observed in a more complex (divergent) SCN under three scenarios of shock in demand. Results reveal that the typology of impulse in customer demand influences SCN performance. In fact, under a combined impulse of customer's demand mean and variability, the effect of the former dominates the latter in terms of demand variability. Meanwhile, an isolated impulse in the 
variability of customer's orders yields the same bullwhip in serial or divergent SCNs. Furthermore, we showed how the Peak of Orders Ratio should be used in conjunction with Order Rate Variance Ratio to measure the bullwhip effect in case of an unexpected and severe alteration of customer demand.

This work reveals which factors of customer demand are needed to be taken into consideration in the event of a severe alteration of such demand. We suggest that managers from either serial or divergent SCNs should not be worried of a sudden increase in customer's demand variability, since it does not have detrimental consequences on the SCN's performance. Nevertheless, this market condition may involve other long-term effects such as the increase of the inventory variance and the increase of the inventory storage capacity. On the contrary, the turbulence caused by a sudden increase in customer's demand mean involves important dynamic consequences, particularly for the divergent SCN and with independence of the current customer's demand variability. In this case, managers of divergent SCNs should pay special attention and try to prevent the detrimental consequences of such scenarios by adopting, for instance, bullwhip-avoidance strategies (i.e. information exchange and/or smoothing order policies).

The limitations of this study produce opportunities for future investigations. First, this work analyses the effect of a shock in demand. However, other typologies of customer demand were not considered in our analysis. Also, this work is limited by the assumption that all echelons of SCNs experience the same lead time and inventory parameters. Further models may focus on different set of parameters and study how these key factors may impact on the sensitiveness of serial and divergent SCNs to shock demands by e.g. further analysing different forecasting methods $[15,16]$.

\section{ACKNOWLEDGEMENTS}

This research was supported by the European Commission/Andalusian Agency of Knowledge (Talentia Marie Skłodowska-Curie Cofund Fellow), by the Portuguese Foundation for Science and Technology [grant SFRH/BPD/108491/2015] and by the Spanish Ministry of Science and Innovation, under project “ADDRESS" [code DPI2013-44461-P/DPI].

\section{REFERENCES}

[1] Wang, X.; Disney, S. M. (2016). The bullwhip effect: Progress, trends and directions, European Journal of Operational Research, Vol. 250, No. 3, 691-701, doi:10.1016/j.ejor.2015.07.022

[2] Dominguez, R.; Cannella, S.; Framinan, J. M. (2015). The impact of the supply chain structure on bullwhip effect, Applied Mathematical Modelling, Vol. 39, No. 23-24, 7309-7325, doi:10.1016/j.apm.2015.03.012

[3] Udenio, M.; Fransoo, J. C.; Peels, R. (2015). Destocking, the bullwhip effect, and the credit crisis: Empirical modeling of supply chain dynamics, International Journal of Production Economics, Vol. 160, 34-46, doi:10.1016/j.ijpe.2014.09.008

[4] Wang, Z.; Wang, X.; Ouyang, Y. (2015). Bounded growth of the bullwhip effect under a class of nonlinear ordering policies, European Journal of Operational Research, Vol. 247, No. 1, 72-82, doi:10.1016/j.ejor.2015.04.047

[5] Dominguez, R.; Framinan, J. M.; Cannella, S. (2014). Serial vs. divergent supply chain networks: a comparative analysis of the bullwhip effect, International Journal of Production Research, Vol. 52, No. 7, 2194-2210, doi:10.1080/00207543.2013.860495

[6] Towill, D. R.; Zhou, L.; Disney, S. M. (2007). Reducing the bullwhip effect: Looking through the appropriate lens, International Journal of Production Economics, Vol. 108, No. 1-2, 444-453, doi:10.1016/j.ijpe.2006.12.024

[7] Cannella, S.; Ciancimino, E. (2010). On the bullwhip avoidance phase: Supply chain collaboration and order smoothing, International Journal of Production Research, Vol. 48, No. 22, 6739-6776, doi: $\underline{10.1080 / 00207540903252308}$ 
[8] Dominguez, R.; Framinan, J. M. (2013). A decision management tool: modelling the order fulfilment process by multi-agent systems, International Journal of Management and Decision Making, Vol. 12, No. 3, 240-258, doi:10.1504/IJMDM.2013.056457

[9] Moreno, J. R.; Mula, J.; Campuzano-Bolarin, F. (2015). Increasing the equity of a flower supply chain by improving order management and supplier selection, International Journal of Simulation Modelling, Vol. 14, No. 2, 201-214, doi:10.2507/IJSIMM14(2)2.284

[10] Diez, M.; Mula, J.; Campuzano-Bolarin, F. (2014). Improvement of a distribution network of a direct sale cosmetics supply chain, International Journal of Simulation Modelling, Vol. 13. No. 1, 16-29, doi:10.2507/IJSIMM13(1)2.245

[11] Chen, F.; Drezner, Z.; Ryan, J. K.; Simchi-Levi, D. (2000). Quantifying the bullwhip effect in a simple supply chain: The impact of forecasting, lead times, and information, Management Science, Vol. 46, No. 3, 436-443, doi:10.1287/mnsc.46.3.436.12069

[12] Dejonckheere, J.; Disney, S. M.; Lambrecht, M. R.; Towill, D. R. (2004). The impact of information enrichment on the Bullwhip effect in supply chains: A control engineering perspective, European Journal of Operational Research, Vol. 153, No. 3, 727-750, doi:10.1016/S0377-2217(02)00808-1

[13] Chatfield, D. C.; Kim, J. G.; Harrison, T. P.; Hayya, J. C. (2004). The bullwhip effect - impact of stochastic lead time, information quality, and information sharing: a simulation study, Production and Operations Management, Vol. 13, No. 4, 340-353, doi:10.1111/j.1937-5956.2004.tb00222.x

[14] Cannella, S.; Barbosa-Póvoa, A. P.; Framinan, J. M.; Relvas, S. (2013). Metrics for bullwhip effect analysis, Journal of the Operational Research Society, Vol. 64, No. 1, 1-16, doi:10.1057/jors.2011.139

[15] Liu, C. H.; Xiong, W. (2015). Modelling and simulation of quality risk forecasting in a supply chain, International Journal of Simulation Modelling, Vol. 14, No. 2, 359-370, doi:10.2507/ IJSIMM14(2)CO10

[16] Li, Q.; Disney, S. M.; Gaalman, G. (2014). Avoiding the bullwhip effect using Damped Trend forecasting and the Order-Up-To replenishment policy, International Journal of Production Economics, Vol. 149, 3-16, doi:10.1016/j.ijpe.2013.11.010 